\title{
Validation in the General Population of the iHealth Track Blood Pressure Monitor for Self-Measurement According to the European Society of Hypertension International Protocol Revision 2010: Descriptive Investigation
}

Victoria Mazoteras-Pardo ${ }^{1 *}, \mathrm{RN}, \mathrm{MSc}$, PhD; Ricardo Becerro-De-Bengoa-Vallejo ${ }^{1 *}$, RN, BSc, MLIS, DPM, PhD, DHL, FFPM RCPS; Marta Elena Losa-Iglesias ${ }^{2^{*}}$, RN, BSc, MSc, DP, PhD; Daniel López-López ${ }^{3^{*}}$, BSc, MSc, DP, PhD; Patricia Palomo-López ${ }^{4 *}, \mathrm{MSc}, \mathrm{DP}, \mathrm{PhD}$; David Rodríguez-Sanz ${ }^{1,5^{*}}, \mathrm{PT}, \mathrm{MSc}, \mathrm{DP}, \mathrm{PhD}$; César Calvo-Lobo ${ }^{6^{*}}$, $\mathrm{PT}, \mathrm{MSc}, \mathrm{PhD}$

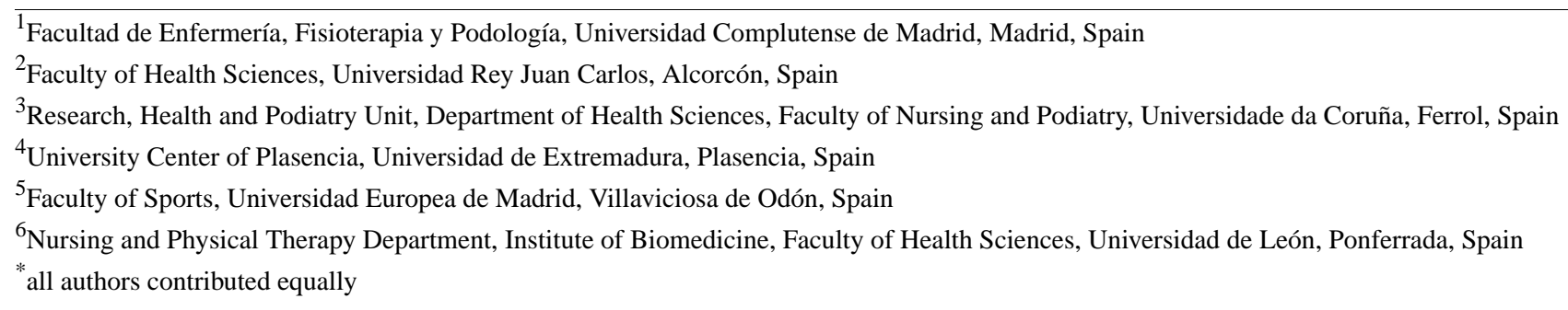

Corresponding Author:

Daniel López-López, BSc, MSc, DP, PhD

Research, Health and Podiatry Unit

Department of Health Sciences, Faculty of Nursing and Podiatry

Universidade da Coruña

Campus Universitario de Esteiro $\mathrm{s} / \mathrm{n}$

Ferrol, 15403

Spain

Phone: 34981337400 ext 3546

Fax: 34981337420

Email: daniel.lopez.lopez@udc.es

\begin{abstract}
Background: High blood pressure is one of the most common reasons why patients seek assistance in daily clinical practice. Screening for hypertension is fundamental and, because hypertension is identified only when blood pressure is measured, accurate measurements are key to the diagnosis and management of this disease. The European Society of Hypertension International Protocol revision 2010 (ESH-IP2) was developed to assess the validity of automatic blood pressure measuring devices that are increasingly being used to replace mercury sphygmomanometers.
\end{abstract}

Objective: We sought to determine whether the iHealth Track blood pressure monitor meets ESH-IP2 requirements for self-measurement of blood pressure and heart rate at the brachial level and is appropriate for use in the general population.

Methods: This study was a descriptive investigation. ESH-IP2 requires a total number of 33 participants. For each measure, the difference between observer and device blood pressure and heart rate values is calculated. In all, 99 pairs of blood pressure differences are classified into 3 categories $(\leq 5, \leq 10$, and $\leq 15 \mathrm{~mm} \mathrm{Hg})$, and 99 pairs of heart rate differences are classified into 3 categories ( $\leq 3, \leq 5$, and $\leq 8$ beats $/ \mathrm{min})$. We followed these protocol procedures in a convenience sample of 33 participants.

Results: iHealth Track fulfilled ESH-IP2 requirements and passed the validation process successfully. We observed an absolute difference within $5 \mathrm{~mm} \mathrm{Hg}$ in 75 of 99 comparisons for systolic blood pressure, 78 of 99 comparisons for diastolic blood pressure, and 89 of 99 comparisons for heart rate. The mean differences between the test and standard readings were 4.19 (SD 4.48) $\mathrm{mm}$ $\mathrm{Hg}$ for systolic blood pressure, 3.74 (SD 4.55) $\mathrm{mm} \mathrm{Hg}$ for diastolic blood pressure, and 1.95 (SD 3.27) beats/min for heart rate. With regard to part 2 of ESH-IP2, we observed a minimum of 2 of 3 measurements within a 5-mm Hg difference in 29 of 33 
participants for systolic blood pressure and 26 of 33 for diastolic blood pressure, and a minimum of 2 of 3 measurements within a 3-beat/min difference in 30 of 33 participants for heart rate.

Conclusions: iHealth Track readings differed from the standard by less than 5, 10, and $15 \mathrm{~mm} \mathrm{Hg}$, fulfilling ESH-IP2 requirements. Consequently, this device is suitable for use in the general population.

(JMIR Mhealth Uhealth 2019;7(3):e13137) doi: 10.2196/13137

\section{KEYWORDS}

blood pressure determination; heart rate determination; validation studies; telemedicine

\section{Introduction}

\section{Background}

High blood pressure (BP) is one of the most common reasons why patients seek assistance in daily clinical practice [1-7]. In addition, patients who have normal BP levels at age 50 years have a $90 \%$ lifetime risk of developing hypertension $[8,9]$. Hypertension is the second most frequent cause of cardiovascular diseases, which are the main cause of morbidity and mortality in the world $[4,8,9]$. Thus, BP maintains a strong, continuous, gradual, consistent, predictive, and independent relationship with the appearance of serious cardiovascular complications, such as peripheral arterial disease, stroke, heart attack, sudden death, or heart failure, or with renal pathologies.

Screening for hypertension is therefore fundamental, and many interventions are available, both pharmacologic and nonpharmacologic, to control such pathology and its consequences $[3,5,6]$. Because hypertension is identified only when BP is measured [10], accurate measurements are key to the diagnosis and management of this disease [11-13].

Mercury sphygmomanometers are being used less and less due to prohibitions against the use of mercury. Automatic BP devices are therefore replacing mercury sphygmomanometers [13-15].

To assess the validity of these automatic BP devices, 3 different protocols are used: that of the Association for the Advancement of Medical Instrumentation (AAMI) [16], that of the British Hypertension Society [17], and that of the Working Group on Blood Pressure Monitoring of the European Society of Hypertension (ESH) [18], which combined the previous protocols. The ESH protocol was revised in 2010 (ESH-IP2) [19] to be more demanding than the previous one. These 3 protocols are for adults in the general population.

\section{Objectives}

The purpose of this study was to validate an automatic monitor that measures BP, iHealth Track, in the general population, following ESH-IP2 [19].

We hypothesized that the iHealth Track for home BP monitoring would show validated measures of BP and heart rate (HR), and would meet the requirements of ESH-IP2 in the general population.

\section{Methods}

\section{Study Design}

This study was a descriptive investigation to validate the iHealth Track device for the measurement of BP in the general population according to ESH-IP2 [19] and the Strengthening the Reporting of Observational Studies in Epidemiology criteria [20].

\section{Ethical Information}

The Clinical Research Ethics Committee of Hospital Clínico San Carlos in Madrid, Spain, approved this study (number 18/504-O_P).

This study complies with the ethical principles of the Declaration of Helsinki [21], including amendments from 2000 to 2013.

All participants were informed about the study and gave written informed consent to participate.

\section{The Devices}

\section{Omron M3 IntelliSense}

The reference device used in the study was the Omron M3 IntelliSense (Omron Healthcare, Inc, Kyoto, Japan), which was validated according to the ESH-IP2 [22]. It consists of an automatic oscillometric sphygmomanometer for the automatic measurement of BP and HR at the arm. The device has 2 available sleeve sizes: a standard size that fits arm circumferences of 22 to $32 \mathrm{~cm}$ and a larger size for arm circumferences of 32 to $42 \mathrm{~cm}$.

\section{iHealth Track}

The device to be validated was the iHealth Track automatic device (KN-550BT; iHealthLabs Europe, Paris, France), which records brachial $\mathrm{BP}$ with the oscillometric method, with a range of pressure of 0 to $300 \mathrm{~mm} \mathrm{Hg}$ (measuring accuracy $\pm 3 \mathrm{~mm}$ $\mathrm{Hg}$ ) and an HR range of 40 to 180 beats/min (measurement accuracy $\pm 5 \%$ ).

The systolic BP (SBP), diastolic BP (DBP), and HR are displayed on a liquid crystal display screen. The device has enough memory for 99 measurements. In addition, this unit can be used with Apple Bluetooth 4.0 devices and certain Android Bluetooth 4.0 mobile phones, by means of an app called Health MyVitals, which means that it allows for storage of BP and HR data in wireless devices connected to an iHealth Track and tracks data graphically and visually. 
Textbox 1. Blood pressure (BP) and heart rate (HR) measurement protocol.

- $\quad$ BPA: entry BP and HR, with the standard device (Omron M3 IntelliSense).

- $\quad$ BPB: device detection of BP and HR with the test instrument (iHealth Track). This measurement is used to determine the correct functioning of the test instrument with the participant and is discarded from further analysis.

- $\quad$ BP1: with the standard device.

- BP2: with the test instrument.

- BP3: with the standard device.

- BP4: with the test instrument.

- BP5: with the standard device.

- BP6: with the test instrument.

- BP7: with the standard device.

The unit weighs approximately $348 \mathrm{~g}$ (batteries and sleeve included). It required 4 AAA batteries with an approximate capacity of 250 measurements. The included standard cuff fits the circumferences of the arm in a range of 22 to $42 \mathrm{~cm}$.

\section{Participants and Recruitment}

We recruited a convenience sample of participants in Plasencia, Spain, from a family medical practice known to one of us.

According to the protocol review [19], we included 33 evaluable participants in the study who met the selection criteria, that is, all the inclusion and none of exclusion criteria. The inclusion criteria were men and women of at least 25 years of age, of whom at least 10 were men and 10 were women. The exclusion criteria were having a sustained arrhythmia, circulatory problems contraindicating the use of the cuff, or being pregnant.

\section{Study Protocol}

The validation team consisted of a single researcher with experience in the measurement of BP.

The measurement room was at a comfortable temperature and without any factors that could influence the measurements, including noise and distractions [18,19].

Each participant reported his or her sex and date of birth. We registered weight, height, and body mass index (BMI, calculated using the Quetelet index, where BMI = weight in kilograms / height in meters squared) and measured the arm circumference to ensure that the cuff size was adequate. Subsequently, the participant relaxed for 10 minutes and 9 consecutive BP measurements were taken on the same arm, with the left arm at heart level, according to ESH-IP2 [18,19]. Measurements were taken alternating the Omron M3 IntelliSense (5 times) and the iHealth Track (4 times), as Textbox 1 outlines.

During measurement, participants remained calm, quiet, sitting, and not moving, with the back straight, keeping the feet on the floor in a parallel position, without crossing the legs. They rested the arm on a flat surface, with the palm of the hand upward and the elbow slightly flexed so that their arm was at the height of the heart. The interval between BP measurements was 30 to 60 seconds [19].
All measurements were made in the same room.

\section{Data Analysis}

We performed statistical analyses using IBM SPSS Statistics, version 19 (IBM Corporation). The results are expressed as mean (SD). According to the normality tests of the Shapiro-Wilk test, we analyzed nonparametric data by the Wilcoxon-Mann-Whitney test and parametric data by means of the Student $t$ test for independent samples. Statistical significance was set at $P<.05$.

We based the accuracy of a device, according to ESH-IP2, on comparisons between the test device (iHealth Track) and the reference device (Omron M3). For each participant, we first compared the device measurements BP2, BP4, and BP6 with the standard measurements $\mathrm{BP} 1, \mathrm{BP} 3$, and BP5, respectively, and then with the standard measurements BP3, BP5, and BP7, respectively. We classified the differences between these 2 devices separately for both SBP and DBP according to whether their values were within 5, 10, or $15 \mathrm{~mm} \mathrm{Hg}$ [19] and, for HR, according to whether their values were within 3,5 , or 8 beats/min.

We analyzed and expressed results according to ESH-IP2 requirements to determine whether the device passed or failed the validation protocol. Part 1 and part 2 of the validation process concern the number of differences in the requested ranges for individual measurements (99 measurements) and for individual participants (33 participants), respectively [19].

We used Bland-Altman plots to represent the relationship of the SBP difference (device reference) and mean SBP (device and reference); DBP difference (device reference) and mean DBP (device and reference); and HR difference (device reference) and mean HR (device and reference).

\section{Results}

\section{Participants}

We screened a total of 33 participants. There were 13 men and 20 women. Table 1 shows their age, weight, height, BMI, and arm circumference, as well as the comparison of these according to sex. 
Table 1. Sociodemographics characteristics of the participants.

\begin{tabular}{llllllll}
\hline Characteristics & \multicolumn{2}{l}{ Total group (N=33) } & Men (n=13) & & \multicolumn{2}{l}{ Women (n=20) } \\
& Mean (SD) & Range & Mean (SD) & Range & Mean (SD) & Range \\
\hline Age (years) & $47.94(17.21)$ & $25-87$ & $45.85(16.66)$ & $30.0-84.0$ & $49.30(17.85)$ & $25.0-87.0$ & $.58^{\mathrm{a}}$ \\
Weight $(\mathrm{kg})$ & $72.45(10.47)$ & $54-92$ & $75.54(9.10)$ & $61.0-90.0$ & $70.45(11.02)$ & $54.0-92.0$ & $.18^{\mathrm{b}}$ \\
Height $(\mathrm{cm})$ & $167.06(5.51)$ & $158.0-178.0$ & $171.0(3.48)$ & $165.0-178.0$ & $165.0(5.16)$ & $158.0-175.0$ & $.001^{\mathrm{b}}$ \\
Body mass index $\left(\mathrm{kg} / \mathrm{m}^{2}\right)$ & $25.88(2.85)$ & $20.32-31.14$ & $25.80(2.43)$ & $22.41-29.39$ & $25.93(3.15)$ & $20.32-31.14$ & $.9^{\mathrm{b}}$ \\
Arm circumference $(\mathrm{mm})$ & $285.76(21.80)$ & $230.0-320.0$ & $293.08(13.76)$ & $260.0-310.0$ & $281.0(24.90)$ & $230.0-320.0$ & $.12^{\mathrm{b}}$ \\
\hline
\end{tabular}

${ }^{\mathrm{a}}$ Nonparametric Wilcoxon-Mann-Whitney test.

${ }^{\mathrm{b}}$ Parametric independent Student $t$ test. $P<.05$ was considered statistically significant, with a confidence interval of $95 \%$.

\section{BP Measurements}

Figure 1 shows the validation results for the iHealth Track BP device according to ESH-IP2. The table shows the numbers of measurements differing from the standard device, Omron M3, by 5,10 , and $15 \mathrm{~mm} \mathrm{Hg}$ or less, for SBP and DBP, according to ESH-IP2 [19]. The mean differences between the standard device and the tested device were 4.19 (SD 4.48) $\mathrm{mm} \mathrm{Hg}$ for SBP and 3.74 (SD 4.55) $\mathrm{mm} \mathrm{Hg}$ for DBP.

These analyses showed an absolute difference within $5 \mathrm{~mm} \mathrm{Hg}$ in 75 of 99 pairs of differences for SBP and in 78 of 99 pairs for DBP (vs at least 73 for SBP and 65 for DBP following ESH-IP2 requirements). We observed an absolute difference within $10 \mathrm{~mm} \mathrm{Hg}$ in 93 of 99 comparisons for SBP and in 89 of 99 comparisons for DBP (vs at least 87 for SBP and 81 for DBP following ESH-IP2 requirements). Additionally, 94 of 99 comparisons exhibited an absolute difference within $15 \mathrm{~mm} \mathrm{Hg}$ for SBP an DBP (vs at least 96 for SBP and 93 for DBP following ESH-IP2 requirements). Therefore, we successfully completed part 1 of the device validation for BP.

For part 2 of ESH-IP2, 29 of 33 individuals had a minimum of 2 of 3 comparisons within a 5-mm $\mathrm{Hg}$ difference for SBP, and 26 of 33 participants met this requirement for DBP (vs at least 24 of 33 participants for SBP and DBP following ESH-IP2 requirements). On the other hand, 3 comparisons exceeded the $5 \mathrm{~mm} \mathrm{Hg}$ requirement for SBP and DBP in 3 of 33 participants (vs a maximum of 3 participants for SBP and DBP following ESH-IP2 requirements). Because these 2 conditions were validated, we successfully completed part 2 of the device validation for BP.
Thus, part 3 of the iHealth Track device validation also was passed, because parts 1 and 2 were both validated for SBP and DBP.

Figure 2 shows the validation results for the iHealth Track HR device according to ESH-IP2. The numbers of measurements differing from the standard Omron M3 device by 3, 5, and 8 beats/min or less are reported for HR. The mean difference between the standard and the tested device was 1.95 (SD 3.27) beats/min.

These analyses showed that 89 of 99 comparisons had an absolute difference within 3 beats/min, 91 of 99 comparisons had an absolute difference within 5 beats/min, and 93 of 99 differences had an absolute difference within 8 beats/min. Therefore, we successfully completed part 1 of the device validation for HR.

For part 2 of ESH-IP2, 30 of 33 individuals had a minimum of 2 of 3 comparisons within a 3 -beats/min difference for HR. On the other hand, none of the 33 participants had 3 differences exceeding 3 beats/min. Because these 2 conditions were validated, we successfully completed part 2 of the device validation for $\mathrm{HR}$.

Thus, part 3 of the iHealth Track device validation was passed, because parts 1 and 2 were both validated for HR.

With these results, the iHealth Track device meets ESH-IP2 validation criteria for both $\mathrm{BP}$ (SBP and DBP) and HR for use in the general population.

These results coincide with the Bland-Altman plots showing the differences in measurements between the iHealth Track device and the Omron M3 for SBP (Figure 3), DBP (Figure 4), and HR (Figure 5). 
Figure 1. Validation results for the iHealth Track blood pressure device according to European Society of Hypertension International Protocol revision 2010 (ESH-IP2). Accuracy is determined by the number differences in these ranges both for individual measurements (Part 1) and for individual subjects (Part 2). To pass, a device must achieve all the minimum pass requirements shown. Pass requirements are as required by the EHS-IP2; achieved are as recorded by the device. DBP: diastolic blood pressure; SBP: systolic blood pressure.

\begin{tabular}{|c|c|c|c|c|c|c|c|}
\hline Part 1 & & $\leq 5 \mathrm{~mm} \mathrm{Hg}$ & $\leq 10 \mathrm{~mm} \mathrm{Hg}$ & $\leq 15 \mathrm{~mm} \mathrm{Hg}$ & Grade 1 & Mean (mm Hg) & $\mathrm{SD}(\mathrm{mm} \mathrm{Hg})$ \\
\hline \multirow[t]{2}{*}{ Pass requirements } & Two of & 73 & 87 & 96 & & & \\
\hline & All of & 65 & 81 & 93 & & & \\
\hline \multirow[t]{2}{*}{ Achieved } & SBP & 75 & 93 & 94 & Pass & 4.19 & 4.48 \\
\hline & DBP & 78 & 89 & 94 & Pass & 3.74 & 4.55 \\
\hline Part 2 & & $2 / 3 \leq 5 \mathrm{~mm} \mathrm{Hg}$ & $0 / 3 \leq 5 \mathrm{~mm} \mathrm{Hg}$ & & Grade 2 & & Grade 3 \\
\hline Pass requirements & & $\geq 24$ & $\leq 3$ & & & & \\
\hline \multirow[t]{2}{*}{ Achieved } & SBP & 29 & 3 & & Pass & Pass & \\
\hline & DBP & 26 & 3 & & Pass & Pass & \\
\hline \multirow[t]{2}{*}{ Part 3} & & & & & & & Result \\
\hline & & & & & & & Pass \\
\hline
\end{tabular}

Figure 2. Validation results for the iHealth Track heart rate (HR) device according to European Society of Hypertension International Protocol revision 2010 (ESH-IP2). Accuracy is determined by the number differences in these ranges both for individual measurements (Part 1) and for individual subjects (Part 2). To pass, a device must achieve all the minimum pass requirements shown. Pass requirements are as required by the EHS-IP2; achieved are as recorded by the device.

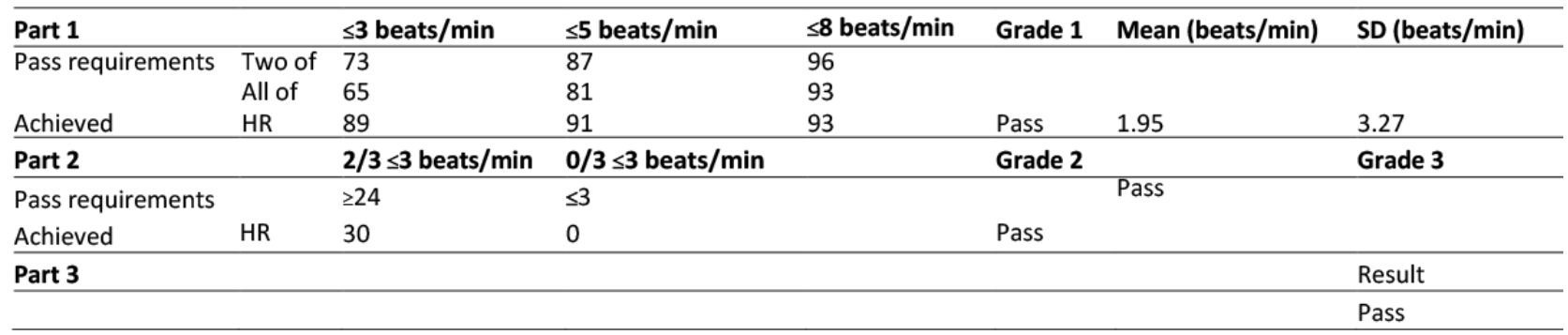

Figure 3. Bland-Altman plot of systolic blood pressure (SBP) measurement differences between the iHealth Track (test) and the Omron M3 (reference) devices in 33 participants. Mean SBP difference is the systolic difference between the devices; mean SBP is the mean systolic average values of the devices.

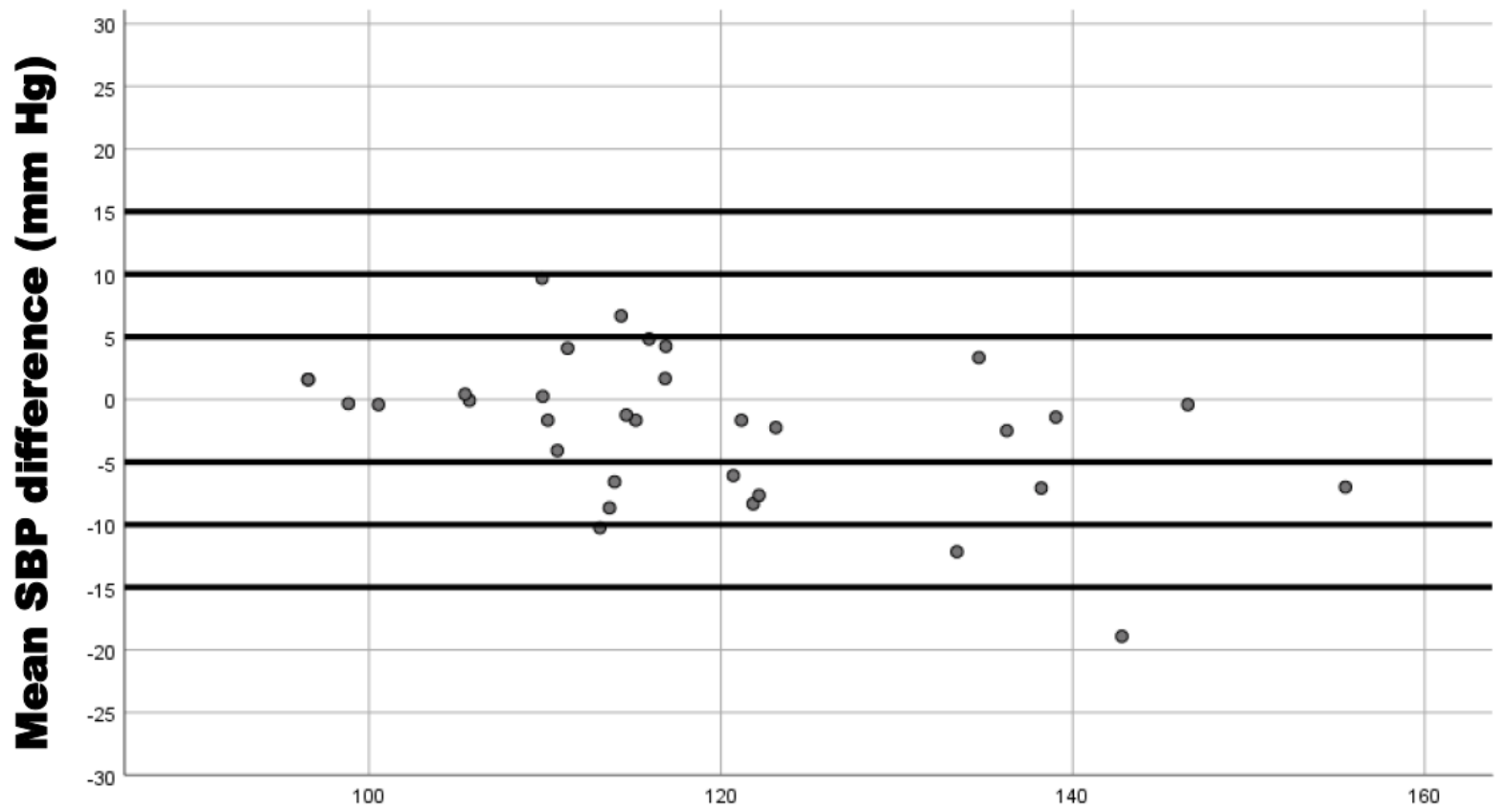

Mean SBP (mm Hg) 
Figure 4. Bland-Altman plot of diastolic blood pressure (DBP) measurement differences between the iHealth Track (test) and the Omron M3 (reference) devices in 33 participants. Mean DBP difference is the diastolic difference between the devices; mean DBP is the mean diastolic average values of the devices.

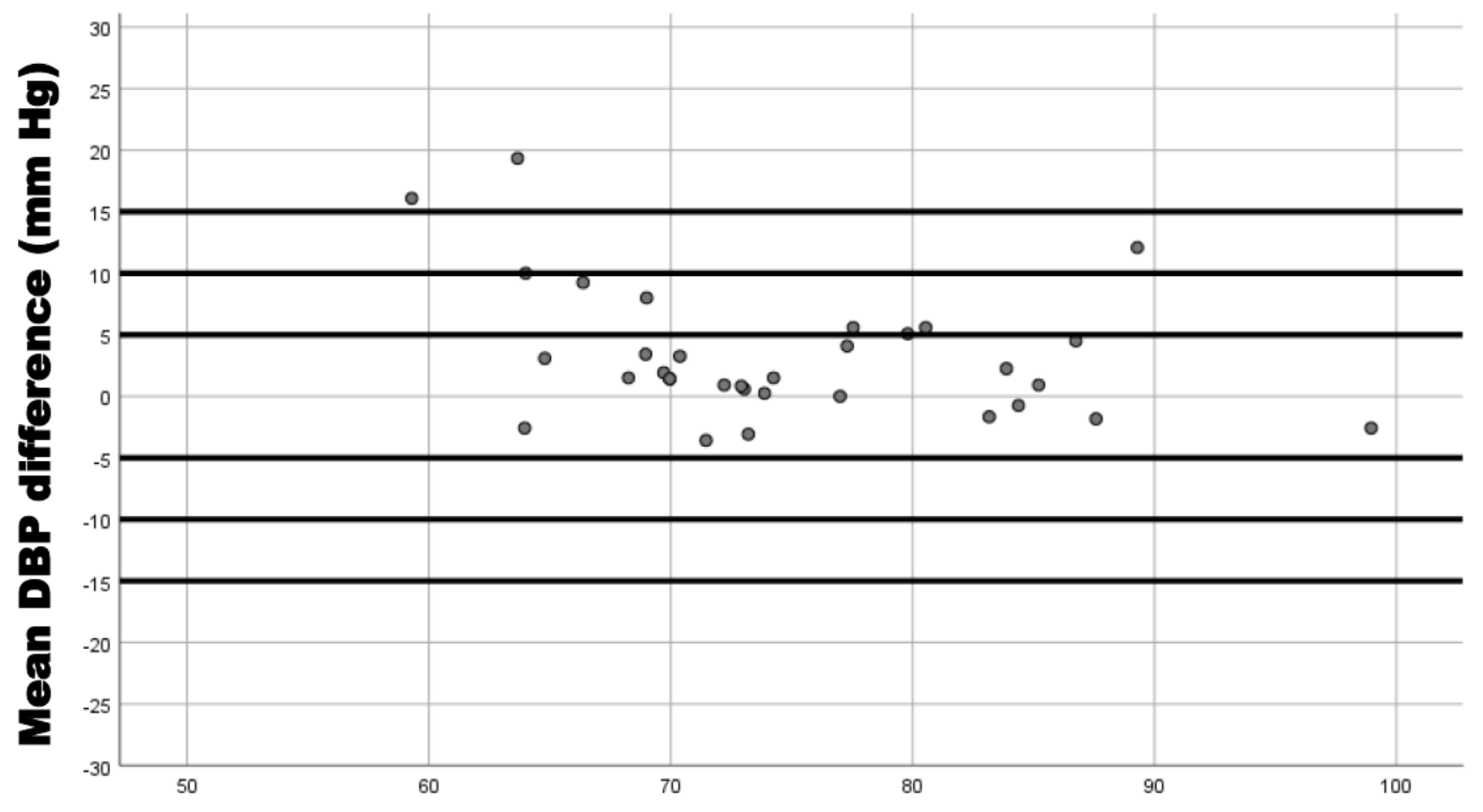

Mean DBP (mm Hg)

Figure 5. Bland-Altman plot of heart rate (HR) measurement differences between the iHealth Track (test) and the Omron M3 (reference) devices in 33 participants. Mean HR difference is the heart rate difference between the devices; mean HR is the mean heart rate average values of the devices.

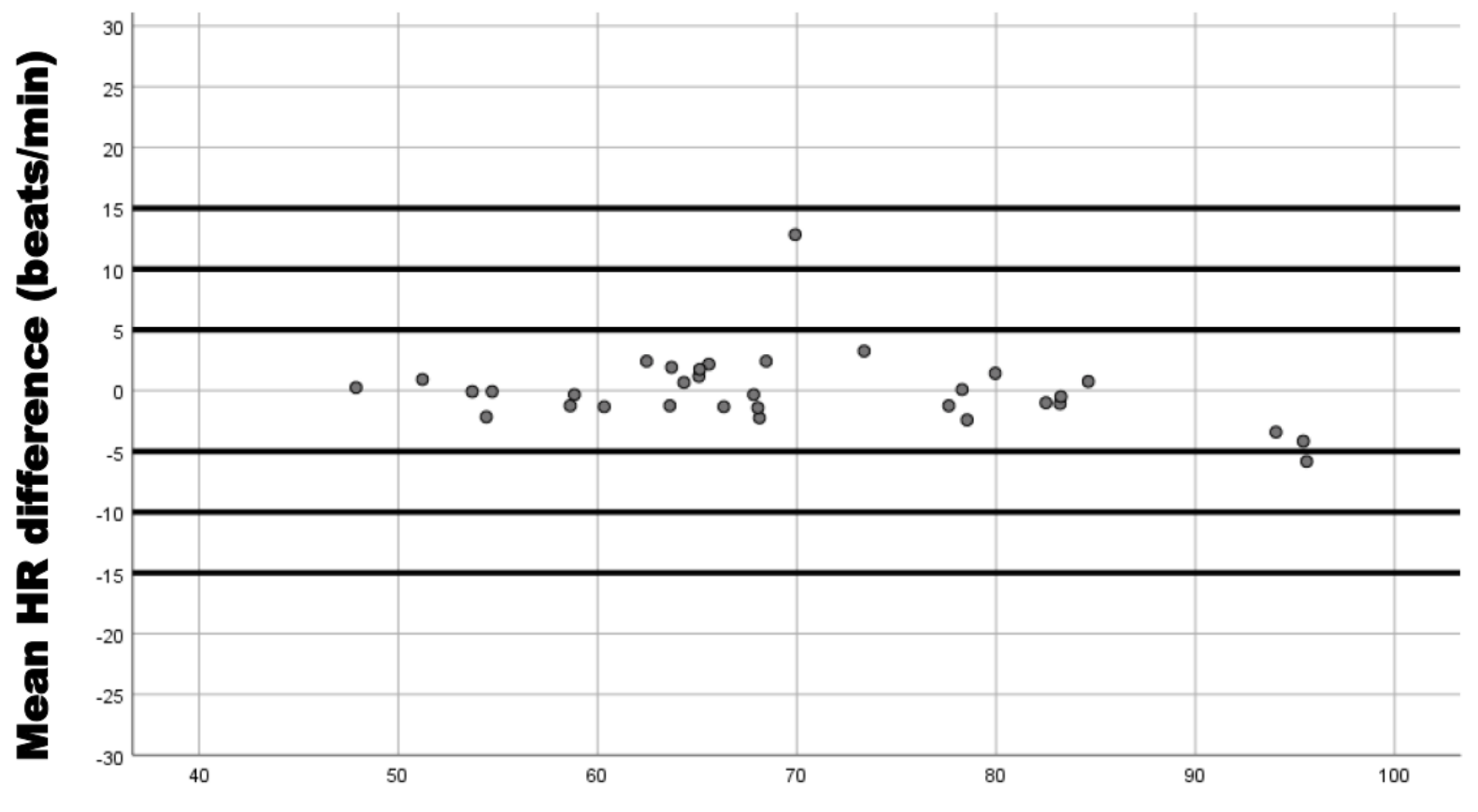

Mean HR (beats/min) 


\section{Discussion}

\section{Principal Results}

The results obtained from this research are important because they are the first to show that the iHealth Track device successfully passed both parts of the validation process according to the ESH-IP2 review in the general population [19]. However, these results cannot be extrapolated to other specific populations, such as older, diabetic, or pregnant individuals, because we have not addressed these conditions.

Regarding the validation protocols, the presence of several protocols for this function [16-19] is problematic for several reasons. Manufacturers cannot perform the 3 protocols and experts focus on their own protocols (eg, the AAMI is followed more in the United States and the ESH is followed in Europe), and it is impossible to compare several validation studies that are governed by different principles [23].

\section{Limitations}

In this study, we used the ESH protocol, first published in 2002 [18] and revised in 2010 [19], which has many advantages over previous ones [16,17], but also has some limitations.

First, ESH-IP2 does not specify the number of validation studies needed to validate the instrument, although a few findings suggest that a device should be validated in no fewer than 2 different centers separately [22-24]. In this regard, the protocol of the AAMI recommends conducting more than 1 study but does not specify the number of studies or devices [16]. Therefore, it is important to check the validity of BP measurement devices before widespread application in clinics and homes.
Second, the specific conditions required for the participants recruited in the study exclude children and young people, thus omitting data on the hypertensive population between 18 and 25 years of age.

Third, although we calculated the sample size, consecutive sampling bias should be considered, and a simple randomization sampling process could be more adequate for future studies.

Fourth, ESH-IP2 mentions no explicit criteria for a validation process in specific populations, and we highly recommended that this be taken into consideration in its next revision.

Fifth, although sphygmomanometers measure SBP, DBP, and $\mathrm{HR}$, no version of the international protocol of the ESH considers validating HR. Hence, we have added such a validation based on the protocol criteria in BP and establishing, in this case, the required differences based on the scale of values found after HR measurements, being even more demanding than the ESH.

\section{Conclusion}

The results of this study are relevant because they show that an automatic wireless device that measures BP and HR and that can also be linked to new technologies meets the requirements of ESH-IP2.

We highly recommended that the accuracy of iHealth Track be assessed in other specific populations, such as pregnant women, older people, patients with arrhythmia, and so on, using other types of sampling. Also, it would be convenient to extend the validation equipment in order to reduce intraobserver error.

\section{Conflicts of Interest}

None declared.

\section{References}

1. Pereira M, Lunet N, Azevedo A, Barros H. Differences in prevalence, awareness, treatment and control of hypertension between developing and developed countries. J Hypertens 2009 May;27(5):963-975. [Medline: 19402221]

2. Banegas JR, Graciani A, de la Cruz-Troca JJ, León-Muñoz LM, Guallar-Castillón P, Coca A, et al. Achievement of cardiometabolic goals in aware hypertensive patients in Spain: a nationwide population-based study. Hypertension 2012 Oct;60(4):898-905. [doi: 10.1161/HYPERTENSIONAHA.112.193078] [Medline: 22949530]

3. Seyedmazhari M. Pharmacological and non-pharmacological treatment of hypertension: a review article. ARYA Atheroscler 2012;8((Special Issue in National Hypertension Treatment):S217-S221 [FREE Full text]

4. Raghu A, Praveen D, Peiris D, Tarassenko L, Clifford G. Engineering a mobile health tool for resource-poor settings to assess and manage cardiovascular disease risk: SMARThealth study. BMC Med Inform Decis Mak 2015;15:36 [FREE Full text] [doi: 10.1186/s12911-015-0148-4] [Medline: 25924825]

5. Chow CK, Teo KK, Rangarajan S, Islam S, Gupta R, Avezum A, et al. Prevalence, awareness, treatment, and control of hypertension in rural and urban communities in high-, middle-, and low-income countries. JAMA 2013 Sep 04;310(9):959-968. [doi: 10.1001/jama.2013.184182] [Medline: 24002282]

6. Khatib R, Schwalm J, Yusuf S, Haynes RB, McKee M, Khan M, et al. Patient and healthcare provider barriers to hypertension awareness, treatment and follow up: a systematic review and meta-analysis of qualitative and quantitative studies. PLoS One 2014;9(1):e84238 [FREE Full text] [doi: 10.1371/journal.pone.0084238] [Medline: 24454721]

7. Bolaji A. Simulation of a real-time mobile health monitoring system model for hypertensive patient in rural Nigeria. Afr J Comput ICT 2014;7(1):95-100 [FREE Full text]

8. Kaplan NM, Victor RG. Hypertension in the population at large. In: Kaplan NM, Victor RG, Flynn JT, editors. Kaplan's Clinical Hypertension. 11th edition. Philadelphia, PA: Wolters Kluwer; 2015:1-17. 
9. James PA, Oparil S, Carter BL, Cushman WC, Dennison-Himmelfarb C, Handler J, et al. 2014 evidence-based guideline for the management of high blood pressure in adults: report from the panel members appointed to the Eighth Joint National Committee (JNC 8). JAMA 2014 Feb 5;311(5):507-520. [doi: 10.1001/jama.2013.284427] [Medline: 24352797]

10. Ogedegbe G, Pickering T. Principles and techniques of blood pressure measurement. Cardiol Clin 2010;28(4):571-586. [doi: 10.1016/j.ccl.2010.07.006] [Medline: 20937442]

11. Daskalopoulou SS, Rabi DM, Zarnke KB, Dasgupta K, Nerenberg K, Cloutier L, et al. The 2015 Canadian Hypertension Education Program recommendations for blood pressure measurement, diagnosis, assessment of risk, prevention, and treatment of hypertension. Can J Cardiol 2015 May;31(5):549-568. [doi: 10.1016/j.cjca.2015.02.016] [Medline: 25936483]

12. Imai Y, Kario K, Shimada K, Kawano Y, Hasebe N, Matsuura H, Japanese Society of Hypertension Committee for Guidelines for Self-monitoring of Blood Pressure at Home. The Japanese Society of Hypertension guidelines for self-monitoring of blood pressure at home (second edition). Hypertens Res 2012 Aug;35(8):777-795. [doi: 10.1038/hr.2012.56] [Medline: $\underline{22863910]}$

13. Mancia G, Fagard R, Narkiewicz K, Redón J, Zanchetti A, Böhm M, Task Force Members. 2013 ESH/ESC Guidelines for the management of arterial hypertension: the Task Force for the management of arterial hypertension of the European Society of Hypertension (ESH) and of the European Society of Cardiology (ESC). J Hypertens 2013 Jul;31(7):1281-1357. [doi: 10.1097/01.hjh.0000431740.32696.cc] [Medline: 23817082]

14. O'Brien E, Asmar R, Beilin L, Imai Y, Mancia G, Mengden T, European Society of Hypertension Working Group on Blood Pressure Monitoring. Practice guidelines of the European Society of Hypertension for clinic, ambulatory and self blood pressure measurement. J Hypertens 2005 Apr;23(4):697-701. [Medline: 15775768]

15. Stergiou GS, Parati G, Asmar R, O'Brien E, European Society of Hypertension Working Group on Blood Pressure Monitoring. Requirements for professional office blood pressure monitors. J Hypertens 2012 Mar;30(3):537-542. [doi:

10.1097/HJH.0b013e32834fcfa5] [Medline: 22241143]

16. Association for the Advancement of Medical Instrumentation. American National Standard for Manual, Electronic, or Automated Sphygmomanometers: ANSI/AAMI SP10-1993. Arlington, VA: AAMI; 1993.

17. O'Brien E, Petrie J, Littler W, de Swiet M, Padfield PL, Altman DG, et al. The British Hypertension Society protocol for the evaluation of blood pressure measuring devices. J Hypertens 1993;11(Suppl 2):S43-S63.

18. O'Brien E, Pickering T, Asmar R, Myers M, Parati G, Staessen J, Working Group on Blood Pressure Monitoring of the European Society of Hypertension. Working Group on Blood Pressure Monitoring of the European Society of Hypertension International Protocol for validation of blood pressure measuring devices in adults. Blood Press Monit 2002 Feb;7(1):3-17. [Medline: $\underline{\text { 12040236] }}$

19. O'Brien E, Atkins N, Stergiou G, Karpettas N, Parati G, Asmar R, Working Group on Blood Pressure Monitoring of the European Society of Hypertension. European Society of Hypertension International Protocol revision 2010 for the validation of blood pressure measuring devices in adults. Blood Press Monit 2010 Feb;15(1):23-38. [doi:

10.1097/MBP.0b013e3283360e98] [Medline: 20110786]

20. Vandenbroucke J, von Elm E, Altman D, Gøtzsche PC, Mulrow CD, Pocock SJ, STROBE Initiative. Strengthening the Reporting of Observational Studies in Epidemiology (STROBE): explanation and elaboration. Int J Surg 2014 Dec;12(12):1500-1524 [FREE Full text] [doi: 10.1016/j.ijsu.2014.07.014] [Medline: 25046751]

21. World Medical Association. WMA Declaration of Helsinki - Ethical Principles for Medical Research Involving Human Subjects. 64th WMA General Assembly, Fortaleza, Brazil, October 2013. Ferney-Voltaire, France: World Medical Association, Inc; 2018 Jul 09. URL: https://www.wma.net/policies-post/ wma-declaration-of-helsinki-ethical-principles-for-medical-research-involving-human-subjects/ [accessed 2019-02-19] [WebCite Cache ID 76JAOgktj]

22. Asmar R, Khabouth J, Topouchian J, El Feghali R, Mattar J. Validation of three automatic devices for self-measurement of blood pressure according to the International Protocol: the Omron M3 Intellisense (HEM-7051-E), the Omron M2 Compact (HEM 7102-E), and the Omron R3-I Plus (HEM 6022-E). Blood Press Monit 2010 Feb;15(1):49-54. [doi: 10.1097/MBP.0b013e3283354b11] [Medline: 20032779]

23. Chahine MN, Topouchian J, Zelveian P, Hakobyan Z, Melkonyan A, Azaki A, et al. Validation of BP devices QardioArm in the general population and Omron M6 Comfort in type II diabetic patients according to the European Society of Hypertension International Protocol (ESH-IP). Med Devices (Auckl) 2018 Dec;11:11-20 [FREE Full text] [doi: 10.2147/MDER.S142126] [Medline: 29343992]

24. Akpolat T, Erdem E, Aydogdu T. Validation of the Omron M3 Intellisense (HEM-7051-E) upper arm blood pressure monitor, for self-measurement, according to the European Society of Hypertension International Protocol revision 2010 in a stage 3-5 chronic kidney disease population. Kidney Blood Press Res 2012;35(2):82-88 [FREE Full text] [doi: 10.1159/000330719] [Medline: 21912183]

\footnotetext{
Abbreviations

AAMI: Association for the Advancement of Medical Instrumentation

BP: blood pressure
} 
DBP: diastolic blood pressure

ESH: European Society of Hypertension

ESH-IP2: European Society of Hypertension International Protocol revision 2010

HR: heart rate

SBP: systolic blood pressure

Edited by G Eysenbach; submitted 14.12.18; peer-reviewed by E Navarro Flores, D Muñoz García; comments to author 02.01.19; revised version received 02.01.19; accepted 03.01.19; published 19.03.19

Please cite as:

Mazoteras-Pardo V, Becerro-De-Bengoa-Vallejo R, Losa-Iglesias ME, López-López D, Palomo-López, P, Rodríguez-Sanz D, Calvo-Lobo C

Validation in the General Population of the iHealth Track Blood Pressure Monitor for Self-Measurement According to the European Society of Hypertension International Protocol Revision 2010: Descriptive Investigation

JMIR Mhealth Uhealth 2019;7(3):e13137

URL: http://mhealth.jmir.org/2019/3/e13137/

doi: $\underline{10.2196 / 13137}$

PMID: 30888331

(C) Victoria Mazoteras-Pardo, Ricardo Becerro-De-Bengoa-Vallejo, Marta Elena Losa-Iglesias, Daniel López-López, Patricia Palomo-López, David Rodríguez-Sanz, César Calvo-Lobo. Originally published in JMIR Mhealth and Uhealth (http://mhealth.jmir.org), 19.03.2019. This is an open-access article distributed under the terms of the Creative Commons Attribution License (https://creativecommons.org/licenses/by/4.0/), which permits unrestricted use, distribution, and reproduction in any medium, provided the original work, first published in JMIR mhealth and uhealth, is properly cited. The complete bibliographic information, a link to the original publication on http://mhealth.jmir.org/, as well as this copyright and license information must be included. 